Originally published as:

Jansen, A., Kleinkauf, N., Weiß, B., Zaiss, N.H., Witte, W., Bornhofen, B., Kist, M., Von EichelStreiber, C., Neumann, M., Michels, H., Eckmanns, T.

Emergence of clostridium difficile ribotype 027 in Germany: Epidemiological and clinical characteristics [Clostridium-difficile-Ribotyp 027: Epidemiologie und Klinik des erstmaligen endemischen Auftretens in Deutschland]

(2010) Zeitschrift fur Gastroenterologie, 48 (9), pp. 1120-1125.

DOI: $10.1055 / \mathrm{s}-0029-1245269$

This is an author manuscript.

The definitive version is available at: https://www.thieme-connect.com/ 


\title{
Clostridium-difficile-Ribotyp 027: Epidemiologie und Klinik des erstmaligen endemischen Auftretens in Deutschland
}

Emergence of Clostridium Difficile Ribotype 027 in Germany: Epidemiological and Clinical Characteristics

\author{
A. Jansen ${ }^{1}$, N. Kleinkauf ${ }^{1}$, B.Weiß ${ }^{1}$, N. H. Zaiss ${ }^{2}$, W.Witte ${ }^{2}$, B. Bornhofen ${ }^{3}$, M. Kist ${ }^{4}$, C. von Eichel- \\ Streiber $^{5}$, M. Neumann ${ }^{6}$, H. Michels ${ }^{7}$, T. Eckmanns $^{1}$ \\ ${ }^{1}$ Robert Koch-Institut, Abteilung für Infektionsepidemiologie, Berlin \\ ${ }^{2}$ Robert Koch-Institut, Fachgebiet 13 - Nosokomiale Infektionen, Wernigerode \\ ${ }^{3}$ Landesuntersuchungsamt Rheinland-Pfalz, Institut für Hygiene und Infektionsschutz, Landau \\ ${ }^{4}$ Konsiliarlabor für Gastrointestinale Infektionen, Abt. für Mikrobiologie und Hygiene, Universität \\ Freiburg \\ ${ }^{5}$ Konsiliarlabor für Clostridium difficile, Institut für Medizinische Mikrobiologie und Hygiene, \\ Universitätsmedizin Mainz, Mainz \\ ${ }^{6}$ Krankenhaus der Barmherzigen Brüder, Trier \\ ${ }^{7}$ Gesundheitsamt der Kreisverwaltung Trier-Saarburg, Trier
}

\section{Zusammenfassung}

Hintergrund: Im September 2007 trat eine Häufung von ungewöhnlich schwer verlaufenden Clostridium- difficile-assoziierten Infektionen (CDI) in einem Trierer Krankenhaus auf. Es wurde vermutet, dass ein neuer Stamm (PCR-Ribotyp 027) mit diesen Ereignissen im Zusammenhang stehen könnte. Zur Untersuchung der Erkrankungsfälle wurde das Gesundheitsamt Trier auf Einladung des Landes Rheinland-Pfalz von einem Feldteam des Robert Koch-Instituts unterstützt. Ziel der Untersuchung war die Aufklärung und Unterbrechung der vermuteten Infektkette.

Methoden: Neben einer retrospektiven Fallsuche von schwer verlaufenden CDI durch Analyse von Patientenakten und Totenscheinen erfolgte eine intensivierte Surveillance von schweren CDI in den Krankenhäusern der betroffenen Region. Dazu wurden bei allen neu auftretenden Verdachtsfällen parallel ein Toxin-A/B-Nachweis und eine selektive Anzucht auf C. difficile durchgeführt. Die Isolatewurden mittels PCR ribotypisiert. Daten zum Krankheitsverlauf und zur Letalität wurden mit einem standardisierten Erhebungsbogen erfasst und statistisch in einer multivariaten Analyse ausgewertet. In dem Index-Krankenhaus wurden Personaluntersuchungen durchgeführt. Ergebnisse: Bis zum 31.1.2008 wurden insgesamt 27 schwere CDI ohne Ribotypisierung und 21 bestätigte Fälle von C.-difficile-Ribotyp-027-Infektionen der Region Trier identifiziert. Im Rahmen der intensivierten Surveillance wurden 399 Patienten untersucht, von denen 76 (19\%) C.-difficile-Isolate angezüchtet werden konnten. Bei 20 Patienten wurde der PCR-Ribotyp 027 nachgewiesen. Insgesamt kam es zu 9 Todesfällen (19\%). Eine bestehende immunsupressive Therapie (Odds Ratio 35,8; 95\%-Konfidenzintervall 2,8 - 464,5) war unabhängiger Risikofaktor für einen letalen Krankheitsverlauf. Schwer verlaufende Infektionen wurden auch bei anderen, Nicht-027-Ribotypen beobachtet. Im Screening vom Krankenhauspersonal des Indexkrankenhauses $(n=161)$ waren $6 \%$ der Mitarbeiter C.-difficile-Toxin positiv.

Diskussion: In dieser Untersuchung konnte erstmalig die endemische Verbreitung von C.-difficilePCR-Ribotyps 027 in einer Region Deutschlands nachgewiesen werden. Als direkte Konsequenz des Ausbruchs wurde Ende 2007 die Ärztliche Meldepflicht für schwer verlaufende CDI eingeführt. Neben krankenhaushygienischen Maßnahmen ist die kritische Verwendung von Antibiotika eine wichtige Maßnahme zur Verhinderung einer weiteren Zunahme von CDI. 


\title{
Einleitung
}

Clostridium (C.) difficile ist die häufigste Ursache nosokomialer, bakteriell bedingter Diarrhö. Das klinische Bild der Clostridium- difficile-assoziierten Erkrankungen („Clostridium Difficile Associated Infections" CDI) reicht von selbstlimitierenden Durchfallerkrankungen bis zur pseudomembranösen Kolitis mit gefürchteten Komplikationen wie toxischem Megakolon und Darmperforation. Der wichtigste prädisponierende Faktor für die Entwicklung einer CDI ist die Antibiotikatherapie, durch die die Zusammensetzung der Darmflora verändert wird. Weitere bekannte Risikofaktoren sind ein höheres Alter, schwere Grunderkrankungen und langer Krankenhausaufenthalt [1, 2].

Seit 2003 wurde aus den USA, Kanada und verschiedenen europäischen Staaten eine Zunahme der Inzidenz von CDI und krankenhausassoziierten Ausbrüchen berichtet. Diese wurden mit dem Auftreten eines neuen Stammes in Verbindung gebracht, der in der PCR als Ribotyp 027, Toxintyp III und in der Pulsfeld-Gelelektrophorese (PFGE) als North American Profile (NAP) 1 charakterisiert wurde [4-6]. Infektionen, die durch diesen Stamm ausgelöst werden, sollen mit einer erhöhten Morbidität und Letalität assoziiert sein [5].

Im September 2007 wurde das Gesundheitsamt (GA) Trier über eine Häufung von schwer verlaufenden C.-difficile-Infektionen auf verschiedenen Stationen eines Trierer Krankenhauses informiert. Innerhalb kurzer Zeit musste bei 3 Patienten aufgrund einer konservativ nicht mehr beherrschbaren pseudomembranösen Kolitis eine Kolektomie durchgeführt werden. Im Oktober 2007 wurde das Robert Koch-Institut vom Land Rheinland-Pfalz zur Unterstützung des GA Trier bei der Untersuchung dieser Fälle eingeladen. Gemeinsam mit dem lokalen Gesundheitsamt und der zuständigen Landesbehörde wurde eine Untersuchung der Fälle begonnen, die die Beschreibung eines möglichen Ausbruchs, die Aufklärung von Infektketten und Interventionen zur Begrenzung neuer Infektionen zum Ziel hatte.

\section{Methoden}

\begin{abstract}
Aktive Fallsuche
Im Rahmen einer prospektiven, intensivierten Surveillance wurde vom 15. Oktober bis zum 30. November 2007 bei allen Patienten der 6 Krankenhäuser des Landkreises Trier-Saarburg und der Stadt Trier sowie einer Rehabilitationsklinik und einem Altenwohnheim der Stadt Trier im Falle eines klinischen Verdachts auf eine CDI mindestens eine Stuhlprobe auf C. difficile untersucht (Toxin A/B und kultureller Nachweis). Der Verdacht auf eine CDI wurde definiert als 1. jede nosokomiale Diarrhö (mit Beginn > $48 \mathrm{~h}$ nach Aufnahme) oder 2. jede ambulant erworbene Diarrhö nach bekanntem stationärem Krankenhausaufenthalt, bekannter Antibiotika-Therapie oder diagnostizierter CDI in der Anamnese der letzten 3 Monaten vor der aktuellen Aufnahme. Ein Verdacht lag definitionsgemäß ebenfalls bei allen Patienten mit toxischem Megakolon oder pseudomembranöser Kolitis vor. Ab November 2007 wurden schwer verlaufende CDI zusätzlich durch eine neu eingeführte Meldepflicht erfasst [9].
\end{abstract}

Ergänzend zur intensivierten Surveillance wurde eine retrospektive Fallsuche eingeleitet. Dazu wurden die Kontaktpatienten aller schwer verlaufenden CDI-Fälle seit Anfang 2007 ermittelt und deren Patientenakten/Arztbriefe auf eine CDI hin überprüft. Zudem wurde die Erwähnung von CDI oder pseudomembranöser Kolitis in Totenscheinen der Jahre 2003 - 2008 untersucht. Daten zum Krankheitsverlauf wurden mit einem standardisierten Erhebungsbogen erfasst.

\section{Falldefinition}

Als bestätigter Fall im Rahmen dieser Untersuchung galt ein Patient, der zwischen dem 1.1.2007 und dem 31.1.2008 im Landkreis Trier-Saarburg oder der Stadt Trier stationär behandelt wurde und folgende Kriterien erfüllte:

- Durchfall (> 3 ungeformte Stühle/24 h) oder

- toxisches Megakolon oder

- pseudomembranöse Kolitis und

- Nachweis eines C.-difficile-Ribotyp-027-Stammes (PCR). Als wahrscheinlicher Fall galt im Rahmen dieser Untersuchung ein Patient, der zwischen dem 1.1.2007 und 31.1.2008 im Landkreis TrierSaarburg oder der Stadt Trier stationär behandelt wurde und folgende Kriterien erfüllte:

- Durchfall oder toxisches Megakolon und 
- C.-difficile-Toxinnachweis oder Nachweis von toxinbildenden C. difficile mit einer anderen Methode (ohne Nachweis eines Ribotypen) oder

- pseudomembranöse Kolitis und mindestens eines der folgenden Kriterien für einen schweren

Verlauf:

- falls nicht nosokomial erworben, die Notwendigkeit der Hospitalisierung aufgrund der Erkrankung,

- die Notwendigkeit einer Wiederaufnahme aufgrund eines Rezidivs,

- Verlegung auf eine Intensivstation zur Behandlung der CDI oder ihrer Komplikationen,

- chirurgischer Eingriff aufgrund eines Megakolons, einer Perforation oder einer refraktären Kolitis,

- Tod < 30 Tage nach Diagnosestellung und CDI als Ursache oder zum Tode beitragende

Erkrankung. Eine ambulant erworbene CDI wurde definiert als eine bereits bei Aufnahme in das

Krankenhaus bzw. in den ersten $48 \mathrm{~h}$ nach Aufnahme manifeste Erkrankung. Schwere Verläufe mit

Nachweis von Nicht-027-Ribotypen wurden in der Auswertung nicht berücksichtigt.

\section{Mikrobiologische Untersuchungen}

C.-difficile-Toxin A/B wurde mittels eines kommerziellen ELISAs (R-Biopharm AG, Darmstadt, Germany) detektiert. Die Anzucht erfolgte auf C.-difficile-selektiven Spezialnährböden unter Zusatz von Cefoxitin und Cycloserin und unter anaeroben Bedingungen (Heipha, Eppelheim, Germany). Bei erfolgreicher Anzüchtung wurde eine Resistenztestung für Erythromycin und Moxifloxacin mittels ETest (AB-Biodisk, Solna, Schweden) durchgeführt. Die PCR-Ribotypisierung wurde wie in der Literatur beschrieben durchgeführt [7].

\section{Personaluntersuchungen}

In einem Krankenhaus wurde auf den Stationen, auf denen Patienten mit schwerer CDI gelegen hatten, freiwillige Stuhl- Untersuchungen auf $C$. difficile bei Ärzten und Pflegepersonal durchgeführt. Zusätzlich wurden Küchenmitarbeiter eines Krankenhauses untersucht.

\section{Statistik}

Odds Ratios (OR) und 95\%-Konfidenzintervalle (95\%-KI) wurden univariat berechnet und durch den X2-Test auf Signifikanz überprüft. Die multivariate Analyse wurde mittels logistischer Regression durchgeführt. Variablen mit $p<0,2$ in der univariaten Analyse wurden in die multivariate Analyse eingeschlossen. Das endgültige Modell wurde mittels eines schrittweisen, rückwärts gerichteten Ausschlussverfahrens unter Berücksichtung möglicher Interaktionsterme ermittelt. Soweit nicht anders angegeben, entsprechen die Werte dem jeweiligen arithmetischen Mittelwert. $p$-Werte $<0,05$ wurden als signifikant gewertet. Die statistische Auswertung der Daten erfolgte mit dem Programm SPSS, v.17 (SPSS, Chicago).

\section{Ergebnisse}

\section{Aktive Fallsuche und mikrobiologische Ergebnisse}

Ingesamt wurden im Rahmen der aktiven Fallsuche 27 wahrscheinliche und 21 bestätigte Fälle von C.-difficile-Ribotyp- 027-Infektionen in 6 Kliniken der Region Trier identifiziert (Abb. 1). Bei der retrospektiven Fallsuche zeigte sich, dass bereits im März 2007 eine CDI mit Nachweis des PCRRibotyps 027 in einem Trierer Krankenhaus aufgetreten war. Hinweise für eine direkte Übertragung von C.-difficile-Ribotyp 027 auf Kontaktpersonen im gleichen Patientenzimmer bestanden in 3 Fällen. In einem Fall lagen für beide Personen Isolate vor, die als Ribotyp 027 identifiziert werden konnten. Die Kontaktpatientin mit Ribotyp 027 war in diesem Fall asymptomatisch. In einem weiteren Fall handelte es sich um die Schwester eines Patienten mit schwerer CDI (ohne Isolat für eine Typisierung), die diesen regelmäßig während seiner CDI-Episoden besucht hatte und dann selber ohne eine bekannte Antibiotika- Vortherapie an CDI erkrankte. Bei ihr konnte der Ribotyp 027 nachgewiesen werden.

Im Rahmen der intensivierten Surveillance wurden 663 Proben von 399 Patienten mit Verdacht auf CDI untersucht. Bei 103 Patienten (26\%) war der C.-difficile A/B-Toxin-ELISA positiv und bei 76 Patienten (19\%) war eine Anzucht erfolgreich. Vier Toxin-negative Proben waren in der Anzucht ebenfalls Kultur-positiv. Von den Isolaten der 76 Kultur-positiven Patienten wurde bei 65 Patienten eine Ribotypisierung durchgeführt (86\%). Der Ribotyp 001 wurde am häufigsten nachgewiesen ( $\mathrm{n}=$ 23; 35\%) (Abb. 2). 20 Patienten wiesen den PCR-Ribotyp 027 auf (31\%). In der Resistenztestung erwiesen sich alle Isolate des PCR-Ribotyps 027 als resistent gegenüber Erythromycin und Moxifloxacin. 
Der Altersmedian der Patienten lag bei $72,5(7-90)$ Jahren. Mit 60\% $(n=29)$ überwog das weibliche Geschlecht. 7 von 48 Infektionen (15\%) wurden ambulant erworben. Bei 13 Patienten (27\%) lag ein Rezidiv der CDI vor. Bei 9 Patienten (19\%) war die CDI die Todesursache oder eine zum Tod kausal beitragende Erkrankung.

Bei den 21 Patienten mit bestätigten PCR-Ribotyp-027-Infektionen lag in 5 Fällen auch ein Kriterium für einen schweren Verlauf vor (3 Patienten wiesen Rezidive auf, ein Patient wurde wegen einer ambulant erworbenen CDI eingewiesen und ein Patient wurde auf die ITS verlegt und verstarb im weiteren Verlauf).

\section{Antibiotikatherapie}

Bei 32 Patienten lagen sichere Angaben zur Antibiotikatherapie vor Beginn der CDI vor. Alle Patienten bis auf einen 7-jährigen Jungen, der mit einer ambulant erworbenen CDI aufgenommen wurde, erhielten in den 3 Monaten vor Beginn der CDI systemische (orale oder parenterale) Antibiotika. Häufigster Vertreter der Cephalosporine war das Ceftriaxon $(n=8)$, bei den Fluorchinolonen das Ciprofloxacin $(n=7)$, daneben Moxifloxacin $(n=2)$ und Levofloxacin $(n=2)$. Unter den Patienten mit Penicillinen (+ Lactamaseinhibitor) hatte die Mehrzahl Ampicillin/Sulbactam $(n=5)$ erhalten. Eine Therapie mit einer Substanzklasse hatten 16 Patienten erhalten, 9 Patienten hatten Antibiotika aus 2 Substanzklassen und 7 Patienten 3 oder mehrere Substanzklassen in den 3 Monaten vor Symptombeginn erhalten.

\section{Risikofaktoren für letale Verläufe}

Eine bestehende immunsupressive Therapie (OR 35,8; 95\% KI 2,8 - 464,5) und die Durchführung einer Kolektomie (OR 39,9; 95\%-KI 2,8 - 576,4) aufgrund der CDI waren in der multivariaten Analyse unabhängige Risikofaktoren für einen letalen Krankheitsverlauf (Tab. 1). Alter und Geschlecht der Patienten sowie weitere untersuchte Faktoren zeigten in der multivariaten Analyse hingegen keinen signifikanten Zusammenhang mit einem letalen Krankheitsverlauf.

\section{Personaluntersuchungen}

Insgesamt wurden 161 Krankenhausmitarbeiter untersucht. Bei 10 Personen war der C.-difficile-ToxinNachweis positiv (6\%): 7 Mitarbeiter von 5 Stationen und 3 Küchenmitarbeiter. C. difficile konnte in keinem Fall kultiviert werden. Zwei der C.-difficile-Toxin-positiven Mitarbeiter waren symptomatisch: eine Küchenmitarbeiterin und eine Krankenschwester. Die Krankenschwester hatte zuvor eine Patientin mit CDI gepflegt und litt über 2 Monate an Durchfällen, bis die Diagnose beim Hausarzt gestellt werden konnte.

\section{Diskussion}

Die hier vorgestellte Untersuchung beschreibt erstmalig das gehäufte Auftreten von C.-difficile-PCRRibotyp 027 in Deutschland im Herbst 2007. Wir konnten zeigen, dass bereits zu diesem Zeitpunkt der C.-difficile-Ribotyp 027 nicht nur auf ein Krankenhaus begrenzt, sondern in der untersuchten Region schon endemisch vorhanden war. Bereits 31\% der untersuchten C.-difficile-Stämme gehörten zum PCR-Ribotyp 027, was ungefähr der Verbreitung dieses Ribotyps in den Niederlanden, Frankreich, Belgien und Luxemburg von $18-27 \%$ entspricht [8]. Da bei der Untersuchung von circa 1000 Isolaten aus Süddeutschland aus dem Zeitraum 2000 - 2006 der neue Stamm nicht gefunden wurde [9], belegen unsere Ergebnisse die eindrucksvolle Fähigkeit des neuen Stammes, sich innerhalb kurzer Zeit regional zu verbreiten. Abgesehen vom C.-difficile-PCRRibotyp 027 zeigte sich eine für Deutschland ansonsten typische Ribotypverteilung mit Prädominanz des Ribotyps 001 und dem Vorkommen von einer Vielzahl anderer Ribotypen in geringer Anzahl.

Der Nachweis des Ribotyps 027 ist dabei nicht zwangsläufig mit einem schweren Krankheitsverlauf assoziiert. In unserer Untersuchung zeigten nur 25\% der CDI durch C.-difficile-Stämme des PCRRibotyps 027 einen nach der Definition schweren Verlauf. Umgekehrt waren schwere CDI auch durch andere Ribotypen verursacht worden. Ein Patient mit einer Ribotyp-001-Infektion musste auf eine Intensivstation verlegt werden, bei jeweils einem Patienten mit einer Ribotyp-001- und -079Infektion verlief die CDI letal. Diese Beobachtungenwerden durch die Ergebnissen einer kürzlich 
veröffentlichten Studie aus Großbritannien unterstützt, die keine erhöhte Morbidität bei Patienten mit Ribotyp-027-Infektionen im Vergleich zu anderen Ribotypen nachweisen konnte [10]. Entsprechend erfasste die in dieser Untersuchung benutzte Definition für wahrscheinliche Ribotyp-027-Fälle möglicherweise auch andere Ribotypen, wobei die mangelnde Spezifität der Definition zugunsten einer hohen Sensitivität in Kauf genommen werden muss.

Insgesamt gibt es in Deutschland zur Verbreitung von CDI nach wie vor nur wenige systematisch erfasste Daten. Als ein Ergebnis der vorliegenden Untersuchung wurde im November 2007 eine bundesweite Meldepflicht für schwer verlaufende C.-difficile-Infektionen etabliert. Die Kriterien für schwere Verläufe orientieren sich dabei weitgehend an der Falldefinition, die auch für diese Untersuchung entwickelt wurde. Es ist jedoch davon auszugehen, dass das tatsächliche Auftreten von schwer verlaufenden CDI über diese Meldepflicht nur unzureichend erfasst wird, da diese nicht laborgebundene Meldepflicht nicht allen Ärzten bekannt ist. Nur in Sachsen existiert seit 2002 eine allgemeine, laborgebundene Meldepflicht für $\mathrm{C}$. difficile. Die Meldedaten aus Sachsen belegen den auch aus anderen europäischen Ländern berichteten dramatischen Anstieg der Inzidenz von CDI [11]. Unterstützt wird dies durch eine Auswertung der ICD-10-codierten Entlassungsdiagnose A04.7 („Enterokolitis durch C. difficile“) des Statistischen Bundesamts [12]. Im Jahr 2008 wurden dem RKI insgesamt 416 Fälle von schwer verlaufender CDI übermittelt, davon 222 Todesfälle. Bei 65 Fällen wurde der Ribotyp 027 nachgewiesen, wobei neben Rheinland-Pfalz und dem Saarland auch Bayern, Hessen, Nordrhein-Westfalen und Baden-Württemberg betroffen waren.

Nahezu alle Patienten dieser Studie wurden vor Beginn der CDI antibiotisch behandelt. Die Verwendung von Cephalosporinen, insbesondere der 2. und 3. Generation, Clindamycin und Penicillinen stellt einen seit langer Zeit bekannten Risikofaktor für die Entwicklung einer CDI dar [2]. Fluorchinolone werden jedoch erst seit der letzten Jahrhundertwende als Risikofaktor angesehen, einhergehend mit einer zunehmenden Verwendung dieser Substanzklasse [13]. Eine Assoziation von CDI mit der Umstellung des Therapieregimes auf neuere Fluorchinolone, wie Moxifloxacin, wurde bereits aus nosokomialen Ausbrüchen berichtet [14]. In einer vor Kurzem veröffentlichten Fallkontrollstudie, die ebenfalls auf den Daten des hier beschriebenen Ausbruchs basiert, wurde eine Antibiotikatherapie mit Fluorchinolonen und Cephalosporinen als der wichtigste unabhängige Risikofaktor für eine C.-difficile-Ribotyp027-Infektion identifiziert [15]. Vor diesem Hintergrund sollte in Deutschland das Konzept der „antibiotic stewardship“ propagiert werden, welches in den USA erfolgreich zur Prävention der CDI eingesetzt wird. Als „antibiotic stewardship“ bezeichnet man dabei Strategien oder Maßnahmen zur Optimierung des Antibiotikaeinsatzes. Eine Leitlinie „Hospital Antibiotic Stewardship“ wird zurzeit von der neu gegründeten Sektion Antibiotic Stewardship der Deutschen Gesellschaft für Infektiologie geplant.

Seltene Komplikationen einer CDI sind die Darmperforation, die Peritonitis und das toxische Megakolon. In solchen schwer verlaufenden Fällen muss ein chirurgisches Vorgehen erwogen werden, und es überrascht daher nicht, dass die Durchführung einer Kolektomie - als ein Indikator für eine solche Komplikation - in unserer Untersuchung mit einer erhöhten Mortalität einherging. In anderen Studien lag die Mortalität von Patienten mit Kolektomie aufgrund einer CDI zwischen 36 $80 \%[16,17]$. Dabei muss man davon ausgehen, dass nicht der operative Eingriff per se zu einer erhöhten Sterblichkeit führt, sondern eher der zu späte Zeitpunkt der chirurgischen Intervention. Im Gegensatz dazu kann eine frühzeitig durchgeführte Kolektomie bei einer schwer verlaufenden CDI zu einer Verringerung der Mortalität führen [18]. Neben einer möglichst frühzeitigen chirurgischen Intervention bei schweren Verläufen scheint auch die Durchführung einer totalen Kolektomie im Vergleich zu anderen Resektionsverfahren einen Überlebensvorteil zu bieten [16]. Auch eine vorbestehende immunsupressive Therapie war in unserer Studie mit einer erhöhten Mortalität belastet. Dies bestätigt die Ergebnisse einer retrospektiven Studie, die gezeigt hat, dass eine Kortikosteroidtherapie bei CDI-Patienten zu einer erhöhten Mortalität führen kann, wobei die Ursachen für diesen Effekt bisher unklar sind [19]. Vor diesem Hintergrund sind Publikationen zum therapeutischen Einsatz von Kortikosteroiden bei CDI kritisch zu bewerten. Jedoch bieten neue Behandlungsstrategien, wie z.B. die allogene Stuhltransplantation oder eine Immunglobulin- Therapie, auch bei schwer verlaufenden CDI vielversprechende therapeutische Optionen [20, 21].

In mehreren der hier untersuchten Fälle war eine Übertragung von C. difficile auf Kontaktpatienten, die im gleichen Zimmer lagen, wahrscheinlich. Dies unterstreicht die Bedeutung von

krankenhaushygienischen Maßnahmen wie insbesondere das Tragen von Handschuhen, sorgfältige Händehygiene, Reinigung und Desinfektion von patientennahen Flächen mit geeigneten Desinfektionsmitteln und eine Isolierung im Einzelzimmer [22]. Der Nachweis von C. difficile bei 
symptomatischem Krankenhauspersonal zeigt, dass bei Durchfällen von medizinischem Personal neben den „klassischen“ darmpathogenen Erregern differenzialdiagnostisch auch C. difficile in Betracht gezogen werden sollte. Auch der zunehmende Nachweis von C. difficile als Ursache ambulant erworbener Durchfall-Erkrankungen sollte in Zukunft diagnostisch berücksichtigt werden [23]. Welche Bedeutung (auch asymptomatisch) infiziertes Personal für die Übertragung von C. difficile im Krankenhaus hat, ist bisher nicht untersucht und sollte Gegenstand zukünftiger Untersuchungen sein.

Zusammenfassend stellt die CDI auch in Deutschland eine zunehmende klinische und gesundheitspolitische Herausforderung dar. Da diese Infektion insbesondere schwerkranke, ältere Patienten betrifft, muss man aufgrund der wachsenden Zahl älterer Menschen in Zukunft mit einem weiteren Anstieg der Inzidenz schwer bzw. letal verlaufender Infektionen rechnen. Eine kontinuierliche Beratung der Stationen bezüglich krankenhaushygienischer Maßnahmen bei C.-difficile-Patienten sowie eine Überprüfung der Umsetzung vor Ort ist zur effektiven Prävention der CDI zwingend notwendig. Antibiotika sollten grundsätzlich restriktiv eingesetzt werden, idealerweise nach Beratung durch Infektiologen und Mikrobiologen bezüglich Indikation und Wahl der Substanzklasse. Schließlich ist eine bundesweite und laborbasierte Surveillance von Infektionen durch C. difficile - unabhängig vom Ribotyp - anzustreben.

\section{Literatur}

1 Bignardi GE. Risk factors for Clostridium difficile infection. The Journal of Hospital Infection 1998; 40: $1-15$

2 Spencer RC. The role of antimicrobial agents in the aetiology of Clostridium difficile-associated disease. The Journal of Antimicrobial Chemotherapy 1998; 41 (Suppl C): 21-27

$3 \mathrm{Kim} \mathrm{KH}$, Fekety R, Batts DH et al. Isolation of Clostridium difficile from the environment and contacts of patients with antibiotic-associated colitis. The Journal of Infectious Diseases 1981; 143: 42-50 4 Kuijper EJ, van den Berg RJ, Debast S et al. Clostridium difficile ribotype 027, toxinotype III, the Netherlands. Emerging Infectious Diseases 2006; 12: 827-830

5 McDonald LC, Killgore GE, Thompson A et al. An epidemic, toxin genevariant strain of Clostridium difficile. The New England Journal of Medicine 2005; 353: 2433-2441

6 Smith A. Outbreak of Clostridium difficile infection in an English hospital linked to hypertoxinproducing strains in Canada and the US. Euro Surveill 2005; 10: E050630.2

7 Borgmann S, Kist M, Jakobiak T et al. Increased number of Clostridium difficile infections and prevalence of Clostridium difficile PCR ribotype 001 in southern Germany. Euro Surveill 2008; 13 (49): 17057

8 Kuijper EJ, Coignard B, Brazier JS et al. Update of Clostridium difficileassociated disease due to PCR ribotype 027 in Europe. Euro Surveill 2007; 12 (6): E1-E2

9 Robert Koch-Institut. Epidemiologisches Bulletin 46/2007 www.rki.de 10 Morgan OW, Rodrigues B, Elston T et al. Clinical severity of Clostridium difficile PCR ribotype 027: a case-case study. PLoS ONE 2008; 3: e1812

11 Burckhardt F, Friedrich A, Beier D et al. Clostridium difficile surveillance trends, Saxony, Germany. Emerging Infectious Diseases 2008; 14: 691-692

12 Vonberg RP, Schwab F, Gastmeier P. Clostridium difficile in discharged inpatients, Germany. Emerging Infectious Diseases 2007; 13 (1): 179- 180

13 Pepin J, Saheb N, Coulombe MA et al. Emergence of fluoroquinolones as the predominant risk factor for Clostridium difficile-associated diarrhea: a cohort study during an epidemic in Quebec. Clin Infect Dis 2005; 41 (9): 1254-1260

14 Biller $P$, Shank $B$, Lind $L$ et al. Moxifloxacin therapy as a risk factor for Clostridium difficileassociated disease during an outbreak: attempts to control a new epidemic strain. Infect Control Hosp Epidemiol 2007; 28: 198-201

15 Weiss B, Kleinkauf N, Eckmanns T et al. Risk factors related to a hospitalassociated cluster of Clostridium difficile PCR ribotype 027 infections in Germany During 2007. Infect Control Hosp Epidemiol 2009; 30: 282-284

16 Hall JF, Berger D. Outcome of colectomy for Clostridium difficile colitis: a plea for early surgical management. Am J Surg 2008; 196 (3): 384- 388

17 Koss K, Clark MA, Sanders DS et al. The outcome of surgery in fulminant Clostridium difficile colitis. Colorectal Dis 2006; 8: 149-154 
18 Seder CW, Villalba Jr MR, Robbins J et al. Early colectomy may be associated with improved survival in fulminant Clostridium difficile colitis: an 8-year experience. Am J Surg 2009; 197 (3): 302307

19 Prendergast TM, Marini CP, D'Angelo AJ et al. Surgical patients with pseudomembranous colitis: factors affecting prognosis. Surgery 1994; 116: 768-774; discussion $774-775$

20 Hedge DD, Strain JD, Heins JR et al. New advances in the treatment of Clostridium difficile infection (CDI). Ther Clin Risk Manag 2008; 4: 949-964

21 Aas J, Gessert CE, Bakken JS. Recurrent Clostridium difficile colitis: case series involving 18 patients treated with donor stool administered via a nasogastric tube. Clin Infect Dis 2003; 36 (5): 580 585, Epub 2003 Feb 14

22 Empfehlungen des Robert Koch-Instituts zu Hygienemaßnahmen bei Patienten mit Durchfällen aufgrund von toxinbildendem Clostridium difficile. Erscheinungsdatum (aktualisierte Version)11.12.2008. http://www.rki.de/cln_100/nn_717602/DE/Content/Infekt/Krankenhaushygiene/ Erreger_ausgewaehlt/Clostridium/Tabelle__Uebersicht. html2007

23 Pituch $\mathrm{H}$. Clostridium difficile is no longer just a nosocomial infection or an infection of adults. Int $\mathrm{J}$ Antimicrob Agents 2009; 33 (Suppl 1): S42-S45

\section{Tabellen und Abbildungen}

Tabelle 1. Ergebnisse der Untersuchung zu Risikofaktoren von letalen Verläufen bei schweren CDI.

\begin{tabular}{|c|c|c|c|c|c|c|}
\hline Faktoren & $\begin{array}{l}\text { Verstorbene } \\
(n=9)\end{array}$ & $\begin{array}{l}\text { Überlebende } \\
(n=45)\end{array}$ & $\begin{array}{l}\text { univariate } \\
\text { OR }\end{array}$ & $\mathbf{p}$ & $\begin{array}{l}\text { multivariate } \\
\text { OR }\end{array}$ & $\mathbf{p}$ \\
\hline Alter (Jahre) & - & - & - & - & $1(0,95-1,05)$ & 1 \\
\hline Geschlecht (männlich) & 3 & 16 & $0,7(0,2-3,3)$ & 1 & & \\
\hline CDI nosokomial erworben & 5 & 32 & $0,3(0,06-1,3)$ & 0,2 & - & n.s. \\
\hline vorbestehende immunsupressive Therapie & 3 & 1 & $19(1,7-214,0)$ & 0,02 & $35,8(2,8-464,5)$ & 0,006 \\
\hline Rezidiv & 2 & 11 & $0,7(0,1-4,1)$ & 1 & & \\
\hline Kolektomie aufgrund der CDI & 3 & 1 & $19(1,7-214,0)$ & 0,02 & $39,9(2,8-576,4)$ & 0,007 \\
\hline SIRS & 4 & 3 & $9,6(1,6-56,1)$ & 0,02 & - & n.s. \\
\hline Verlegung auf ITS & 5 & 13 & $2,5(0,6-10,9)$ & 0,3 & & \\
\hline Pseudomembranöse Kolitis & 7 & 19 & $3,7(0,7-20,0)$ & 0,15 & - & n.s. \\
\hline Transplantation & 1 & 0 & n.d. & n.d. & & \\
\hline
\end{tabular}


Abbildung 1. PCR-Ribotyp-027-Infektionen in Kliniken des Landkreises Trier-Saarburg und der Stadt Trier, 2007. Die schraffierten Felder stellen wahrscheinliche $(n=22)$, die ausgefüllten Felder bestätigte PCR-Ribotyp-027-Infektionen $(n=21)$ dar.

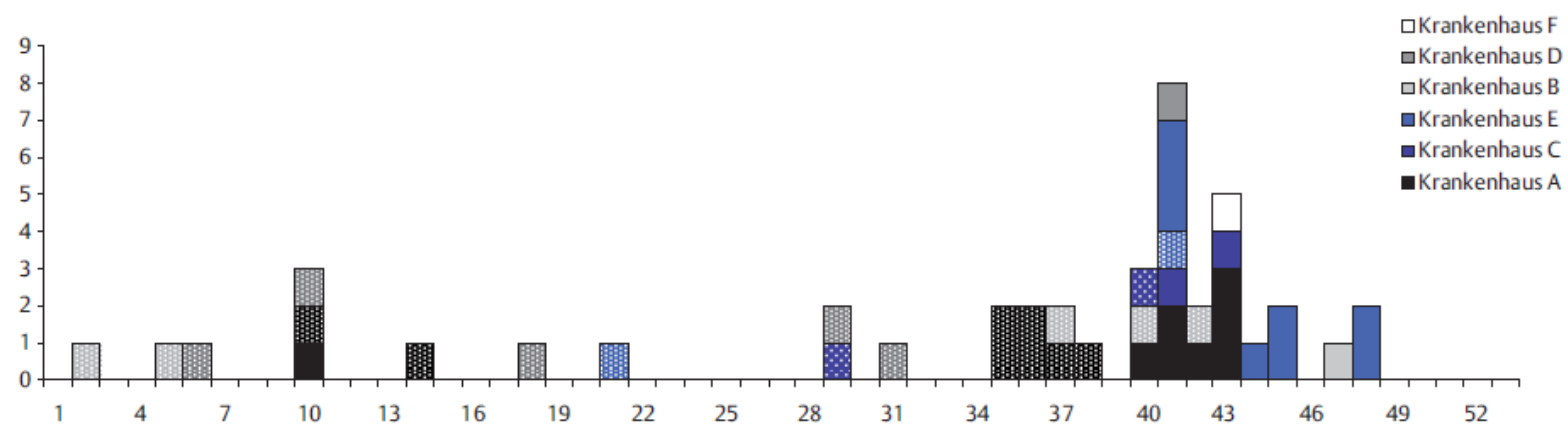

Abbildung 2. C.-difficile-PCR-Ribotyp-Verteilung im Landkreis Trier-Saarburg und der Stadt Trier, Ergebnisse der intensivierten Surveillance in 6 Krankenhäusern, einer Rehabilitations-Klinik und einem Altenwohnheim, 15.10. - 30.11.2007 ( $n=65)$.

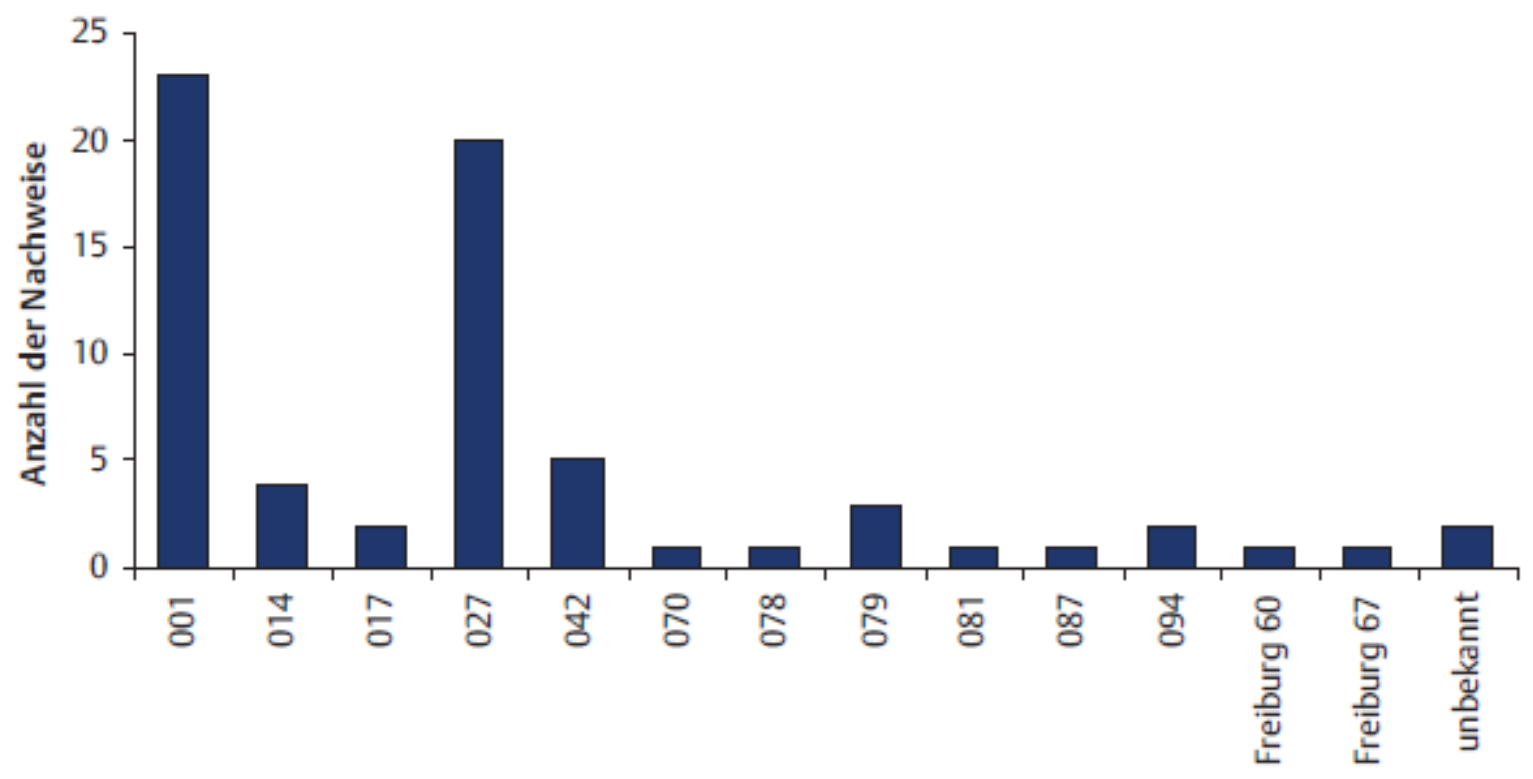

PCR-Ribotyp 